\title{
Téoros
}

Revue de recherche en tourisme

\section{Tunnel sous la Manche et tourisme : l'Euro-Région sort du tunnel}

\section{Pascal Thérain}

Volume 8, numéro 1, mars 1989

France-Québec

URI : https://id.erudit.org/iderudit/1080351ar

DOI : https://doi.org/10.7202/1080351ar

Aller au sommaire du numéro

Éditeur(s)

Université du Québec à Montréal

ISSN

0712-8657 (imprimé)

1923-2705 (numérique)

Découvrir la revue

Citer cet article

Thérain, P. (1989). Tunnel sous la Manche et tourisme : l'Euro-Région sort du tunnel. Téoros, 8(1), 14-15. https://doi.org/10.7202/1080351ar d'utilisation que vous pouvez consulter en ligne.

https://apropos.erudit.org/fr/usagers/politique-dutilisation/ 


\section{Tunnel sous la Manche et tourisme:

L'acte unique européen, la mise en service du tunnel sous la Manche et du TGV-Nord, la réalisation de nouvelles infrastructures routières et autoroutières vont modifier profondément le paysage touristique du NordPas de Calais.

Chacun se prend à rêver: les projets et initiatives se multiplient, tous plus intéressants les uns que les autres, tous plus ambiticux.

Et pourtant, les responsables politiques et touristiques gardent la tête froide. Ils savent bien, en effet, que ce futur contexte régional et européen, s'il est porteur de nombreux espoirs, ne permettra pas de multiplier à l'infini les opportunités de développement touristique. Ils savent également que le préalable à ce développement est l'élaboration d'une véritable stratégie définissant les priorités en matière d'équipement, de promotion et de formation.

C'est pourquoi le Conseil Régional Nord-Pas de Calais a confié à son Comité Régional de Tourisme le soin de définir au travers du Schéma Régional de Développement Touristique ses grandes orientations et priorités pour les cinq années à venir. Celles-ci ont été largement prises en compte dans l'élaboration du futur contrat de dixième plan et du troisième plan régional.

\section{La stratégie régionale de développement touristique}

Mettre en oeuvre une politique d'offre de produits et d'animations attractifs, s'appuyant sur le développement d'équipements d'accueil de qualité, accompagné d'un haut niveau de service, tels sont les trois axes prioritaires de cette stratégie destinée à attirer, accueillir et retenir les touristes dans le Nord-Pas de Calais.

Cette région ne pouvant miser sur son climat pour affirmer son image de marque touristique, priorité sera donnée au développement de produits touristiques indépendants de ces contraintes, mobilisant les efforts des prestataires vers des formules "quatre saisons" de courts et moyens séjours.

Trois programmes d'action pour la mise en oeuvre de cette stratégie:

* Pascal Thérain est Directeur du CRT du Nord-Pas de Calais
- l'aménagement concerté de pôles de développement touristique;

- le développement de filières touristiques prioritaires;

- la modernisation et l'organisation de l'offre touristique.

\section{La notion de pôles prioritaires} de développement

Le phénomène touristique ne concerne pas l'ensemble du territoire régional.

En effet, le développement touristique régional, pour être significatif, doit s'appuyer sur des zones susceptibles de constituer à terme de véritables pôles structurants de l'activité touristique, capables d'attirer, de retenir et de fidéliser, non seulement la clientèle régionale mais aussi l'ensemble des nouveaux marchés européens.

Si le Nord-Pas de Calais est globalement positionné sur le marché du loisir de proximite, seules quelques zones ont la vocation et la capacité de développer une véritable activité économique touristique basće sur le court séjour. Il s'agit en l'occurrence du littoral, de son avant-pays et de l'Avesnois. Ces deux pôles devront définir leur développement en fonction de leur vocation actuelle, de leurs clientèles mais surtout des concepts marketing qui en constituent les points forts et les produits d'appel. Ainsi le littoral NordPas de Calais pourra valoriser ses stations et son avant-pays au travers des concepts de culture de la mer, de pays aux activités multiples ou d'espace... L'Avesnois jouera la carte du tourisme vert, des arts et traditions populaires ou des loisirs actifs.

\section{La notion de filières touristiques prioritaires}

Afin de contribuer à la mise en valeur d'autres zones géographiques, hors littoral et Avesnois, aux richesses naturelles, patrimoniales et culturelles importantes, sera favorisée la création de produits touristiques de journées ou de courts séjours s'articulant autour de filières (tourisme de nature, tourisme de santé, remise en forme, tourisme culturel).

\section{Seront ainsi développées:}

- La découverte de la nature, principalement dans les zones du parc naturel régional qui bénéficieront d'équipements touristiques légers, d'une signalisation adaptée, d'outils d'information et de sensibilisation du public.
- Les activités de plein air, au travers d'activités corporelles et sportives telles que la pếche, les randonnées, le golf, les sports de glisse, etc... Des produits hautde-gamme et compétitifs seront élaborés en tenant compte des besoins des clientèles nord-européennes et de la concurrence étrangère.

- Le tourisme de santé et de remise en forme; celui-ci s'appuiera sur deux produits essentiels: le thermalisme marin (thalassotherapie au Touquet, centre de remise en forme à Wissant et Berck...) et la station thermale de Saint-Amand.

- Le tourisme culturel: par des actions de valorisation du patrimoine monumental, muséographique, industriel et événementiel. Ce produit prendra largement en compte la dimension interrégionale (Picardie....) ou euro-régionale (Kent, Flandres belges...).

Le développement de ces filières nécessitera la mise en place d'un partenariat technique et financier, public et privé, et une programmation des projets à cinq ans.

\section{La modernisation et I'organisa- tion de l'offre touristique}

La modernisation de l'offre touristique du Nord-Pas de Calais et son organisation constituent enfin un élément indispensable de valorisation des équipements et des produits touristiques qui seront élaborés dans le cadre du futur marché européen.

Quatre priorités ont été retenues par les responsables politiques et techniques du NordPas de Calais:

- Favoriser la prospection, la commercialisation et la mise en marché du tourisme régional par l'actualisation de la connaissance des clientèles des marchés prioritaires ciblés depuis deux ans et la définition d'une stratégic de production, de commercialisation et de communication sur ces pays.

- Rénover et moderniser l'hébergement en particulier sur les zones prioritaires de développement touristique ou celles faisant l'objet d'un contrat de filière.

- Favoriser l'ouverture internationale du territoire par:

La mise en oeuvre d'un schéma régional de signalisation touristique en cohérence avec les expériences de nos voisins du Kent et de la Belgique. 
- La mise en place d'un réseau de points "accueil, information, services" devant permettre aux touristes - et ce de façon permanente (terminal $\mathrm{GB}$ et français du tunnel, gare TGV) ou saisonnière (postes frontières autoroutiers, gares, ports) - de les informer grấce à des outils informatisés permettant le choix et la réservation des differents produits touristiques régionaux.

La sensibilisation et la formation des acteurs du tourisme

Dans ce futur contexte européen très concurrentiel:

- Le tourisme régional n'echappera pas a l'exigence d'une qualité d'accueil et d'un professionnalisme toujours accru qui implique que soit renforcée et développée l'offre en matière de., formation initiale (en matière de management et d'ingénierie touristique),.. formation continue notamment dans le domaine des langues vivantes, des techniques d'accueil et de commercialisation et des technologies nouvelles appliqueses au tourisme.

Ces actions seront completées par une démarche plus générale de promotion du territoire régional auprès de ses propres habitants et notamment des jeunes.

\section{L'euro-région}

Depuis de nombreuses années, la région Nord-Pas de Calais s'est engagée très fortement dans une politique de cooperation transfrontalière, que ce soit avec le Kent en Angleterre ou avec la Flandre et la Wallonie en Belgique. Celle-ci se traduit d'ores et déjà par des actions de promotion communes et une collaboration régulière en matière d'aménagement touristique. Ainsi, les responsables du Comité Régional de Tourisme du Nord-Pas de Calais et leurs homologues du Kent ou du Westtoerism se rencontrentils régulièrement afin de préparer diverses initiatives communes et de s'informer de leurs projets respectifs. Dans ect esprit, 1'association Nuances qui regroupe le CRT, les deux CDT et divers professionnels du tourisme régional Nord-Pas de Calais) et le Kent Cruisader, véritable tour-opérateur régional du Comté de Kent, ont réalisé un catalogue commun de produits courts séjours, associant la découverte des musées, des monuments, à celle des sites naturels les plus remarquables de ces deux régions.

\section{En conclusion}

Avec ses 10 milliards de chiffre d'affaires annuel, ses 45000 emplois directs, ses 14 millions de touristes étrangers européens qui transitent chaque annee par ses frontières, un marché potentiel de 35 millions de personnes résidant à moins de 3 heures de route, le Nord-Pas de Calais peut afficher de réelles ambitions en matière de tourisme.

Demain, graice au tunnel et au TGV, il va pouvoir s'ouvrir sur un nouveau marché tou. ristique de plus de 90 millions d'individus.

Charte de conpération pour la promotion et le développement entre la région Nord-Pas de Calais et le Comté de Kent.

Le Conseil Regional Nord-Pus de Calais et le Kent Country Council, conscients de leurs relations par rapport à leur situation de "porte d'entrée" et en anticipant les effets du tunnel sous la Manche, ont décidé d'etendre la coopération régionale à la promotion et uu développement de leur potentiel touristique.

Par conséquent, ils adoptent la Charte de cooperation dejà existante entre le Conseils des districts de Canterbury, Douvres. Thanet et Shepway, dans le Kent, et le Parc naturel régional du Boulonnais, dans le Nord-Pas de Calais..

(Ils, s'engagent à):

- Assurer la promotion commune des deux entites géographiques;

- Encourager la collaboration des professionnels français et britanniques de façon à améliorer leur connaissance des produits touristiques existants sur chacun des marchts ef les aider à elaborer des produits communs;

- Commercialiser ces nouveaux produits touristiques en France, en Grande-Bretagne et sur les marchés Eirangers.:

- Promouvoir des initiatives communes et concertées vis-à-vis de la CEE, de Eurotunnel en des gouvernements français et anglais pour assurer la défense légitimes dés régions concernées.

Un "Comite" de pilotage est constitué, composé de 12 membres (6 par nationalité à raison de 2 élus, 2 professionnels et de 2 techniciens du tourisme)...

Noell Josephe, Président du Conseil Régional Nord-Pas de Calais et

Tony Hart, Leader Kent Country Council.

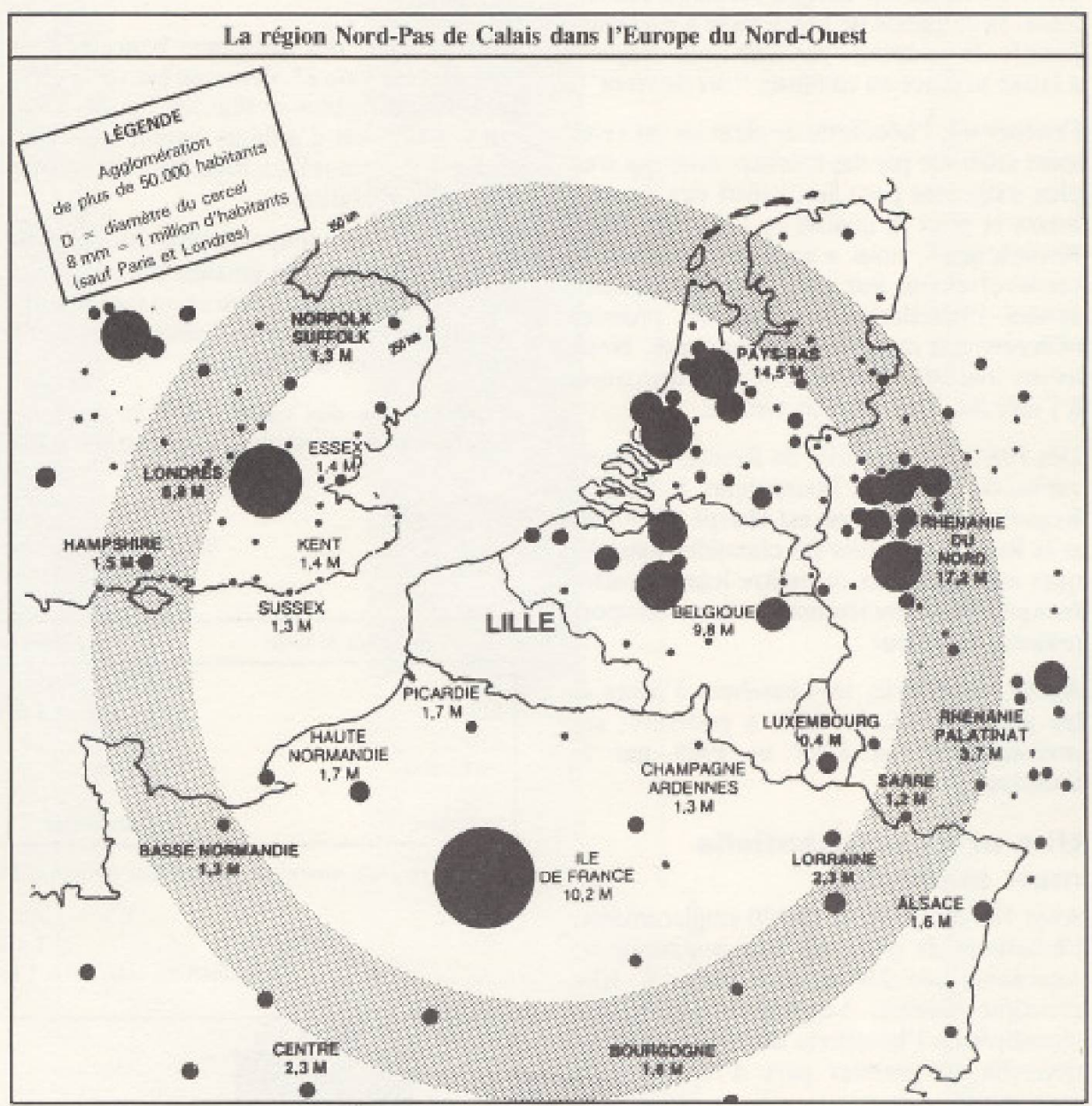

Saura-t-il profité de cette fabuleuse opportunité qui lui est offerte? Sera-t-il capable de se tailler une part avantageuse du gẩteau européen? Chacun - élu, technicien, professionnel, investisseur - en a l'ambition.

L'heure est au partenariat, à l'imagination mais aussi à la rigueur des choix et des priorités. Le Schéma Régional de Développe- ment Touristique et la stratégie décrite cidessus constituent sans aucun doute, dans leur application, un premier pas sur le chemin de la réussite.

Le consensus entre tous les acteurs régionaux du tourisme et la poursuite d'un partenariat effectif et imaginatif avec nos régions et pays voisins en sont la condition. 